\title{
Вклад распределенного эффекта Пельтье в эффективность ветви термоэлектрического охладителя
}

\author{
(C) О.И. Марков \\ Орловский государственный университет, \\ 302026 Орел, Россия \\ E-mail: O.I.Markov@mail.ru
}

Поступила в Редакцию 19 сентября 2021 г.

В окончательной редакции 24 сентября 2021 г. Принята к публикации 24 сентября 2021 г.

\begin{abstract}
Сделана попытка расчета вклада распределенного эффекта Пельтье в эффективность ветви термоэлемента $Z$ при различных видах распределения примесей. С этой целью численными методами решалась граничная задача теплового баланса в ветви термоэлемента с учетом распределенного эффекта Пельтье. Рассматривался случай невырожденных носителей заряда в рамках стандартной двухзонной модели. Параметры носителей заряда подбирались близкими термоэлектрикам на основе теллуридов висмута и сурьмы. Как показал расчет в рамках двухзонной модели, использование распределенного эффекта Пельтье приводит лишь к частичному поглощению теплоты Джоуля, что способствует росту общей эффективности ветви. При этом параметр $Z$ вдоль значительной части ветви принимает величины, существенно меньшие максимального значения.
\end{abstract}

Ключевые слова: термоэлемент, эффект Пельтье, параметр термоэлектричекой эффективности, стандартная двухзонная модель.

DOI: 10.21883/FTP.2022.01.51811.27

\section{1. Введение}

Идея использования переменного состава термоэлектриков для повышения эффективности преобразования термоэлектрического устройства, работающего в широком диапазоне температур, была первоначально предложена А.Ф. Иоффе [1]. Существуют по крайней мере две возможности повышения эффективности: с помощью переменной концентрации носителей заряда: оптимизация параметра эффективности $Z$ в каждой точке ветви в рабочем интервале температуры [2] и использование распределенного эффекта Пельтье [3]. Применение распределенного эффекта Пельтье считается одним из перспективных путей повышения эффективности термоэлектриков. Реализация этой идеи заключает в себе ряд трудностей, связанных с математическим моделированием, определением оптимального распределения концентрации носителей заряда, приводящего к максимальной эффективности ветви, изготовлением ветви с оптимальным распределением концентрации носителей заряда. Так как практически использующиеся термоэлектрики - это легированные полупроводники $n$ - и p-типа, то обычно при оптимизации рассматриваются только основные носители заряда. Предполагается, что их концентрацию можно изменять с помощью легирования соответствующими примесями в очень широком интервале концентраций примеси, не учитывая собственных носителей заряда. При этом возникает соблазн полностью компенсировать теплоту Джоуля с помощью распределенного эффекта Пельтье $[3,4]$. Как показано в работе [3], в оптимизированной ветви с полной компенсацией теплоты Джоуля электропроводность на холодном конце ветви на 2 порядка больше, чем на горячем, а модуль дифференциальной термоэдс в 4 раза меньше. В работе [5] для поддержания оптимального значения $Z$ в PbTe также используют носители только одного знака - электроны, концентрация которых определяется концентрацией легирующей примеси. В работе [6] отмечается, что материалы на основе $\mathrm{Bi}_{2} \mathrm{Te}_{3}$ при большом перепаде температур в ветви термоэлемента обладают существенно меньшим, чем его максимальное значение, общим параметром эффективности. Параметр эффективности, близкий к максимуму, в каждой точке ветви рекомендовано поддерживать изменением состава материала по длине ветви термоэлемента. В обзоре [7] указывается, что определение оптимального профиля неоднородности примеси является сложной математической задачей, перечисляются некоторые методы расчета температурного профиля ветви термоэлемента и рассматриваются технологии создания неоднородностей.

Реальные низкотемпературные термоэлектрики с высокой эффективностью не обладают широкой запрещенной зоной, поэтому необходимо учитывать и собственные носители заряда $[8,9]$. В данной работе на основе решения граничной задачи теплового баланса с использованием классической статистики сделана попытка расчета вклада распределенного эффекта Пельтье в эффективность ветви термоэлемента на базе двухзонной модели при различных видах распределения примесей вдоль ветви. Так как при непосредственном вычислении параметра эффективности $Z$ вклад эффекта Томсона не учитывается, то этот эффект в решении граничной задачи также не рассматривался.

\section{2. Методика расчета}

В целях упрощения решения задачи ограничимся случаем невырожденных носителей заряда и простей- 
шей стандартной зонной моделью. Чтобы результаты расчета приблизить к реальности, зонные параметры соответствуют параметрам низкотемпературного термоэлектрика на основе теллуридов висмута и сурьмы: ширина запрещенной зоны $E_{g}=0.15$ эВ, эффективные массы плотности состояний электронов $m_{c}^{*}=0.45 m_{0}$, дырок $m_{v}^{*}=0.69 m_{0}[10]$.

В классическом приближении концентрация электронов и дырок определяются выражениями [11]

$$
\begin{aligned}
& n=2\left(\frac{2 \pi m_{c}^{*} k T}{h^{2}}\right)^{3 / 2} e^{\eta_{e}}=N_{c} e^{\eta_{e}}, \\
& p=2\left(\frac{2 \pi m_{v}^{*} k T}{h^{2}}\right)^{3 / 2} e^{\eta_{h}}=N_{v} e^{\eta_{h}},
\end{aligned}
$$

где $N_{c}, N_{v}$ - эффективное число состояний зоны проводимости и валентной зоны соответственно, $\eta_{e}, \eta_{h}-$ приведенный химический потенциал электронов и дырок соответственно. Концентрации электронов и дырок связаны уравнением электронейтральности

$$
n=p+N_{d},
$$

где $N_{d}$ - концентрация донорной примеси. Подразумевается, что все атомы примеси ионизованы.

Удельные электропроводности электронов $\sigma_{n}=e U_{n} n$ и дырок $\sigma_{p}=e U_{P} p$ в сумме определяют полную удельную электропроводность

$$
\sigma=\sigma_{n}+\sigma_{p}
$$

Соответствие результатов расчета кинетических коэффициентов с экспериментом можно улучшить, учитывая температурные зависимости подвижностей носителей заряда в виде $U_{n}=a T^{-3 / 2}, U_{p}=b T^{-1.8}[10,12]$, где $a=617.3 \mathrm{~m}^{2} \mathrm{~K}^{1.5} / \mathrm{B} \cdot \mathrm{c}, \quad b=1449 \mathrm{M}^{2} \mathrm{~K}^{1.5} / \mathrm{B} \cdot \mathrm{c}$. Концентрационные зависимости подвижностей электронов и дырок не учитывались. Удельная теплопроводность полупроводника [11]

$$
\chi=\chi_{\mathrm{ph}}+\left(\frac{k}{e}\right)^{2} T\left[2 \sigma+\frac{\sigma_{n} \sigma_{p}}{\sigma}\left(\frac{E_{g}}{k T}+4\right)^{2}\right],
$$

где $\chi_{\mathrm{ph}}-$ фононная теплопроводность учтена в виде зависимости $\chi_{\mathrm{ph}}=c T^{-1}[9]$, с коэффициентом $c=235 \mathrm{BT} / \mathrm{M}$.

Дифференциальная термоэдс электронов [10]

$$
\alpha_{n}=-\frac{k}{e}\left(r+2-\eta_{e}\right),
$$

где $r=0$ (рассеяние на акустических фононах), приведенный химический потенциал электронов определялся по формуле

$$
\eta_{e}=\ln \frac{n}{N_{c}} .
$$

Дифференциальная термоэдс дырок

$$
\alpha_{p}=\frac{k}{e}\left(r+2-\eta_{h}\right)
$$

где приведенный химический потенциал дырок

$$
\eta_{h}=-\left(\frac{E_{g}}{k T}+\eta_{e}\right) .
$$

Полная дифференциальная термоэдс

$$
\alpha=\frac{\alpha_{p} \sigma_{p}+\alpha_{n} \sigma_{n}}{\sigma_{p}+\sigma_{n}} .
$$

Расчет параметра эффективности $Z=\alpha^{2} \rho \chi$ согласно приведенным формулам при $T=300 \mathrm{~K}$ показывает, что максимальное значение $3 \cdot 10^{-3} \mathrm{~K}^{-1}$ достигается при значении химического потенциала $\mu=-0.009$ эВ и концентрации примеси $N_{d}=5 \cdot 10^{24} \mathrm{M}^{=3}$. Согласно элементарной теории, такая эффективность позволяет достичь минимальной температуры $T_{c}=225 \mathrm{~K}$. При этой темпеpaтуре $Z=2.95 \cdot 10^{-3} \mathrm{~K}^{-1}$, оптимальные $\mu=-0.01$ эВ и концентрация $N_{d}=3 \cdot 10^{24} \mathrm{M}^{-3}$. Естественно, возникает вопрос об оптимальной концентрации примеси в однородной ветви. Ответ можно получить при решении граничной задачи теплового баланса

$$
\frac{d}{d \xi}\left(\chi \frac{d T}{d \xi}\right)+\frac{Y^{2}}{\sigma}=0
$$

с граничными условиями

$$
\left.\chi \frac{d T}{d \xi}\right|_{\xi=0}=\left.\alpha Y T\right|_{\xi=0},\left.T\right|_{\xi=1}=T_{1},
$$

где $\xi=x / l(0<\xi<1), Y=I l / S$ - параметр оптимизации [13], $l$ - длина ветви, $S$ - сечение ветви, $I-$ ток ветви.

Так как граничная задача $(11),(12)$ нелинейная, то ее решение возможно численными методами. Минимизацию температуры холодного конца ветви необходимо проводить по параметру $Y$ и по концентрации примеси $N_{d}$. В режиме максимального перепада температур оптимальная концентрация для однородной ветви оказалась равной $N_{d}=3.3 \cdot 10^{24} \mathrm{M}^{-3}$, минимально достижимая температура на холодном конце составляла $230 \mathrm{~K}$. Рассчитанное распределение температуры вдоль однородной ветви отражает кривая 1 (рис. 1), которая, судя по кривой 1 (рис. 2) градиента температуры, достаточно близка к параболе. При постоянной концентрации примеси параметр эффективности $Z$ не остается постоянным вдоль ветви (рис. 3, кривая 1), сначала он незначительно растет, затем немного снижается к горячему концу ветви. Химический потенциал также понижается с ростом температуры (рис. 4, кривая 1) до -0.02 эВ. Отсюда следует, что однородная ветвь термоэлемента не может быть оптимальной по параметру эффективности на всей длине ветви во всем рабочем интервале температур и для оптимизации $Z$ нужно использовать неоднородную по составу ветвь.

Для расчета распределения примеси неоднородной ветви нужно использовать граничную задачу теплового 


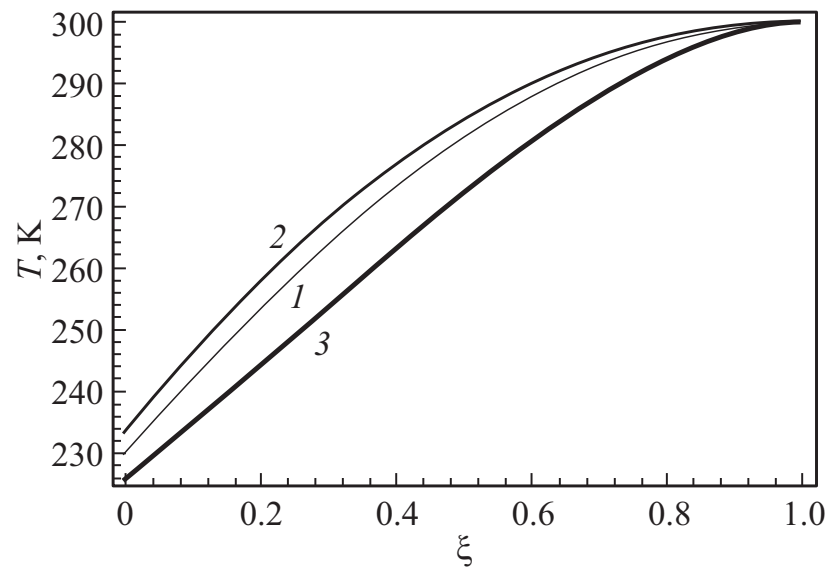

Рис. 1. Распределение температуры вдоль: однородной ветви с оптимальной концентрацией примеси $N_{d}=3.3 \cdot 10^{24} \mathrm{M}^{-3}$ (кривая 1), неоднородной ветви с концентрациями $N_{d}(0)=3 \cdot 10^{24} \mathrm{M}^{-3} \quad$ и $\quad N_{d}(1)=5 \cdot 10^{24} \mathrm{M}^{-3} \quad($ кривая 2), неоднородной ветви с концентрациями $N_{d}(0)=5 \cdot 10^{24} \mathrm{M}^{-3}$ и $N_{d}(1)=1.3 \cdot 10^{24} \mathrm{M}^{-3}$ (кривая 3).

баланса с учетом распределенного эффекта Пельтье:

$$
\frac{d}{d \xi}\left(\chi \frac{d T}{d \xi}\right)+\frac{Y^{2}}{\sigma}-Y T \frac{d \alpha}{d n} \frac{d n}{d \xi}=0
$$

с прежними граничными условиями (12). Так как имеется аналитическая зависимость между концентрацией электронов и концентрацией примеси

$$
n\left(N_{d}\right)=\frac{N_{d}}{2}\left(\sqrt{1+\frac{4 n_{i}^{2}}{N_{d}^{2}}}+1\right),
$$

производную от концентрации электронов можно выразить через производную от концентрации примеси

$$
\frac{d n}{d \xi}=\frac{d n}{d N_{d}} \frac{d N_{d}}{d \xi},
$$

что позволяет решать граничную задачу (12), (13) при различных вариантах распределения примеси вдоль ветви $N_{d}(\xi)$.

Можно предположить, что повысить параметр термоэлектрической эффективности возможно распределением концентрации примеси в пределах от оптимального значения $N_{d}(0)=3 \cdot 10^{24} \mathrm{M}^{-3}$ на холодном конце до оптимального значения $N_{d}(1)=5 \cdot 10^{24} \mathrm{M}^{-3}$ на горячем. В предположении линейной зависимости концентрации примеси между указанными значениями решение граничной задачи (12), (13) с одновременной оптимизацией по параметру $Y$ показало, что вместо ожидаемого понижения минимально достижимой температуры на холодном конце ветви она повышается до $234 \mathrm{~K}$ (рис. 1 , кривая 2), Кривая зависимости температуры становится параболической, о чем можно судить по прямолинейной зависимости градиента температуры (рис. 2, кривая 2). Небольшое повышение градиента температуры вблизи холодного конца ветви по сравнению с предыдущим случаем приводит к повышению минимальной температуры, т.е. уменьшению эффективности ветви в целом. Расчет параметра эффективности $Z$ показывает, что линейное распределение примеси вблизи холодного конца ветви дает значения, практически совпадающие с однородным распределением, а затем позволяет поднять его по всей длине ветви почти до максимального значения (рис. 3, кривая 2). Этот результат, состоящий в том, что параметр эффективности повысился, а эффективность ветви в целом упала, мог бы показаться парадоксальным, если не учитывать выделение теплоты распределенного эффекта Пельтье. Таким образом, использование

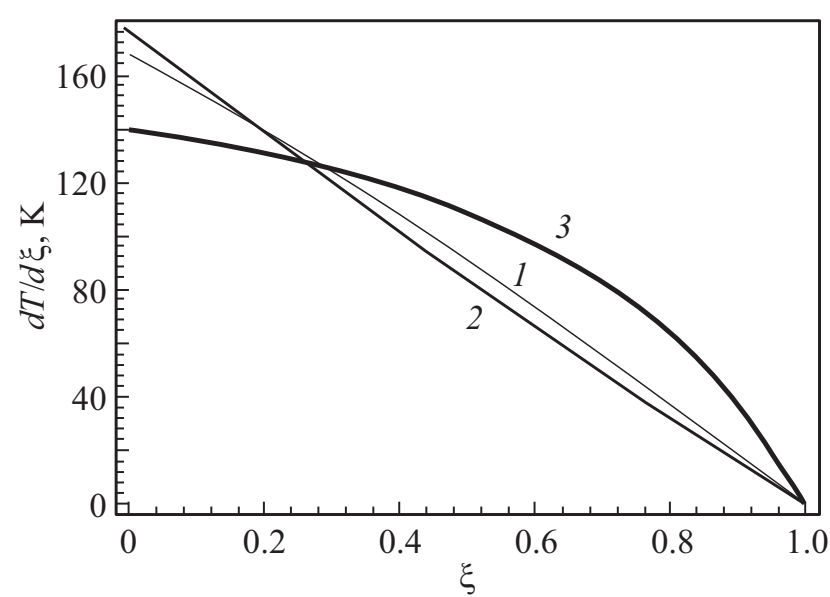

Рис. 2. Распределение градиента температуры вдоль: однородной ветви с оптимальной концентрацией примеси $N_{d}=3.3 \cdot 10^{24} \mathrm{M}^{-3} \quad$ (кривая 1$)$, неоднородной ветви концентрациями $N_{d}(0)=3 \cdot 10^{24} \mathrm{M}^{-3}$ и $N_{d}(1)=5 \cdot 10^{24} \mathrm{M}^{-3}$ (кривая 2), неоднородной ветви с концентрациями $N_{d}(0)=5 \cdot 10^{24} \mathrm{M}^{-3}$ и $N_{d}(1)=1.3 \cdot 10^{24} \mathrm{M}^{-3}$ (кривая 3).

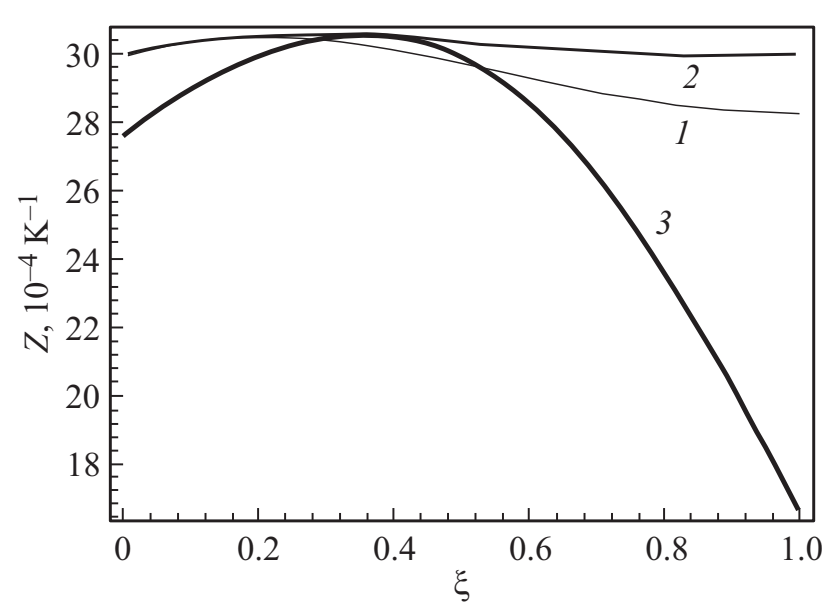

Рис. 3. Распределение параметра эффективности вдоль: однородной ветви с оптимальной концентрацией примеси $N_{d}=3.3 \cdot 10^{24} \mathrm{M}^{-3} \quad$ (кривая 1$)$, неоднородной ветви с концентрациями $N_{d}(0)=3 \cdot 10^{24} \mathrm{M}^{-3}$ и $N_{d}(1)=5 \cdot 10^{24} \mathrm{M}^{-3}$ (кривая 2), неоднородной ветви с концентрациями $N_{d}(0)=5 \cdot 10^{24} \mathrm{M}^{-3}$ и $N_{d}(1)=1.3 \cdot 10^{24} \mathrm{M}^{-3}$ (кривая 3). 


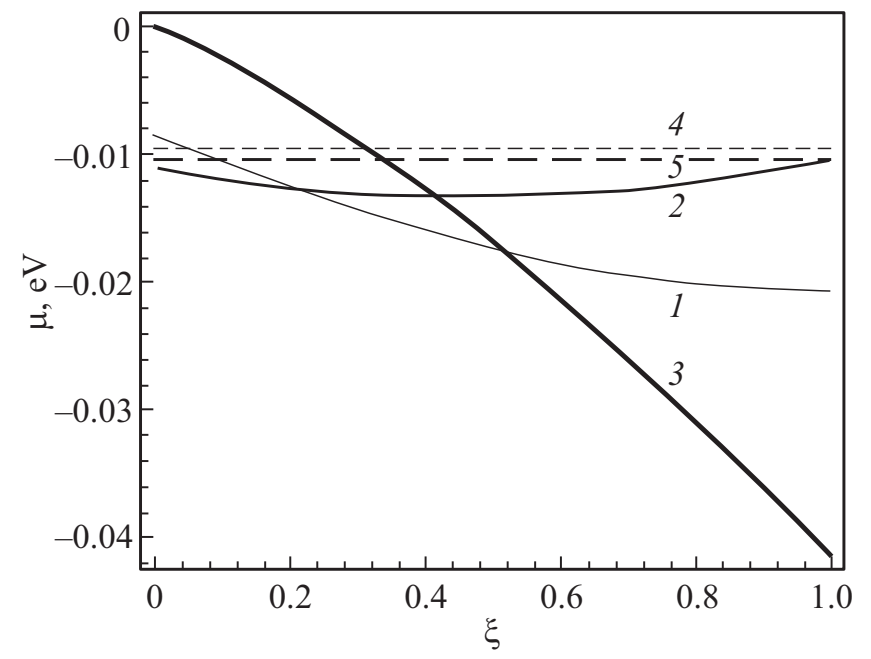

Рис. 4. Распределение химического потенциала вдоль: однородной ветви с оптимальной концентрацией примеси $N_{d}=3.3 \cdot 10^{24} \mathrm{M}^{-3} \quad$ (кривая 1$)$, неоднородной ветви с концентрациями $N_{d}(0)=3 \cdot 10^{24} \mathrm{M}^{-3}$ и $N_{d}(1)=5 \cdot 10^{24} \mathrm{M}^{-3}$ (кривая 2), неоднородной ветви с концентрациями $N_{d}(0)=5 \cdot 10^{24} \mathrm{M}^{-3}$ и $N_{d}(1)=1.3 \cdot 10^{24} \mathrm{M}^{-3} \quad$ (кривая 3). Штриховые 4 и 5 отвечают оптимальным значениям химического потенциала при 300 и $225 \mathrm{~K}$ соответственно.

неоднородной ветви с линейным ростом концентрации примеси позволяет повысить параметр эффективности $Z$ по всей длине ветви, но распределенное тепловыделение Пельтье приводит к тому, что эффективность ветви в целом снижается. То, что это именно так, подтверждается еще тем, что расчет при уменьшенном интервале концентраций до $N_{d}(0)=4 \cdot 10^{24} \mathrm{M}^{-3}$ и $N_{d}(1)=5 \cdot 10^{24} \mathrm{M}^{-3}$ показывает снижение температуры до $233 \mathrm{~K}$, т. е. повышение эффективности ветви в целом. Очевидно, что это вызвано уменьшением распределенного эффекта Пельтье. Расчет химического потенциала показывает (рис. 4, кривая 2), что химический потенциал снижен до -0.013 эВ в центральной части ветви.

Повышение эффективности можно ожидать при смене градиента концентрации примеси на обратный. При этом для увеличения распределенного эффекта Пельтье необходимо максимально увеличить область роста модуля дифференциальной термоэдс, что обеспечивается бо́льшим интервалом изменения донорной примеси. При этом очевидно, что некоторая часть ветви неизбежно будет иметь показатель эффективности $Z$ существенно ниже его максимального значения. Расширение интервала концентраций со стороны больших величин ограничено падением модуля термоэдс из-за вырождения носителей заряда, а нижняя граница концентрации электронов связана с максимальным значением модуля дифференциальной термоэдс, которое возникает с ростом собственной концентрации носителей заряда, что можно учесть только в рамках двухзонной модели. Выбор интервала изменения концентраций примеси был сделан после решения серии граничных задач (12), (13) при различных значениях концентрации примеси на холодном конце ветви. Оказалось, что максимальное снижение температуры возможно в пределах линейного распределения концентраций примеси от $N_{d}(0)=5 \cdot 10^{24} \mathrm{M}^{-3}$ на холодном конце до $N_{d}(1)=1.3 \cdot 10^{24} \mathrm{M}^{-3}$ на горячем. Кривая распределения температуры 3 (рис. 1) становится более пологой, а кривая градиента температуры 3 (рис. 2) показывает, что вблизи холодного конца ветви он существенно уменьшается по сравнению с предыдущими случаями (рис. 2, кривые 1,2). Это приводит к тому, что минимально достижимая температура на холодном конце при таком распределении примеси составляет 226 K. Интересно, что на холодном и горячем концах показатель эффективности (рис. 3, кривая 3) существенно меньше максимального $Z$, а среднее значение по длине ветви составляет $2.7 \cdot 10^{-3} \mathrm{~K}^{-1}$. Максимальное значение параметра $Z$ принимает в той области ветви, где кривая химического потенциала (рис. 4, кривая 3) пересекает „коридор эффективности“ $-(0.009-0.01)$ эВ (кривые 4 и 5). Таким образом, повышение эффективности термоэлектрического преобразования энергии с использованием распределенного эффекта Пельтье не сводится только к увеличению параметра эффективности $Z$, но еще требуется и максимально расширить область изменения концентрации основных носителей заряда.

Таким образом, в режиме максимального перепада температуры модуль дифференциальной термоэдс от холодного конца к горячему увеличивается в 1.5 раза, а удельное сопротивление в 4.8 , что существенно отличается от данных расчетов для однозонной модели термоэлектрика [4]. Поэтому в рамках более реалистичной двухзонной модели возможна лишь частичная компенсация теплоты Джоуля с помощью распределенного эффекта Пельтье [9]. Из результатов проведенных расчетов следует, что снижение температуры холодного спая по отношению к видам распределения примеси не столь критично, поэтому вместо поиска оптимального распределения примеси вдоль ветви проще подбирать распределение, которое проще осуществить технологически.

\section{3. Заключение}

Проведенное исследование позволяет заключить, что в рамках двухзонной модели:

- однородная по составу ветвь термоэлемента в режиме максимального перепада температуры не может иметь одинаково высокий параметр эффективности $Z$ по всей длине ветви термоэлемента;

- оптимизация ветви в соответствии с максимальными значениями $Z$ на холодном и горячем концах и линейным распределением концентрации носителей заряда не приводит к повышению ее эффективности изза тепловыделения в распределенном эффекте Пельтье; 
- распределенный эффект Пельтье можно использовать для частичной компенсации теплоты Джоуля и перераспределения теплового баланса вдоль ветви термоэлемента, что повышает эффективность преобразования энергии при снижении средней величины параметра эффективности $Z$.

\section{Конфликт интересов}

Автор заявляет, что у него нет конфликта интересов.

\section{Список литературы}

[1] А.Ф. Иоффе. Полупроводниковые термоэлементы (М.-Л., АН СССР, 1960).

[2] Е.К. Иорданишвили. ФТТ, 8 (10), 3118 (1966).

[3] Ю.И. Агеев, К.Ф. Иванова, М.А. Каганов, Л.С. Стильбанс, Э.М. Шер. ЖТФ, 55 (11), 2266 (1985).

[4] К.Ф. Иванова, А.С. Ривкин. ЖТФ, 52 (7), 1406 (1982).

[5] S. Dashevsky, S. Shusterman, M.P. Dariel, I. Drabkin. J. Appl. Phys., 92 (3), 1425 (2002).

[6] A.E. Kaliazin, V.L. Kuznetsov, D.M. Rowe. Proc. 20th Int. Conf. on Thermoelectrics, Beijing, China, June, 8-I 1 (IEEE, Piscataway, NJ, 2001) p. 286.

[7] V.L. Kuznetsov. Functionally Graded Materials for Thermoelectric Applications, In book: CRC Thermoelectrics Handbook: Macro to Nano, ed. by D.M. Rowe (CRC Press, Taylor \& Francis Group, Boca Raton, London-N.Y., 2006) chap. 38 .

[8] О.И. Марков. Докл. ХІІІ Межгос. сем. „Термоэлектрики и их применения“, 2013, с. 180.

[9] О.И. Марков. ЖТФ, 91 (11), 1722 (2021).

[10] М. Гольцман, В.А. Кудинов, И.А. Смирнов. Полупроводниковые термоэлектрические материалы на основе $\mathrm{Bi}_{2} \mathrm{Te}_{3}$ (М., Наука, 1972).

[11] Б.И. Аскеров. Электронные явления переноса в полупроводниках (М., Наука, 1985).

[12] Термоэлектрические охладители, под ред. А.Л. Вайнера (М., Радио и связь, 1983).

[13] О.И. Марков. ЖТФ, 75 (6), 132 (2005).

\section{Contribution of the distributed Peltier effect to the efficiency of the thermoelectric cooler branch}

\section{O.I. Markov}

Orel State University named after I.S. Turgenev, 302026 Orel, Russia

Abstract In this paper, an attempt is made to calculate the contribution of the distributed Peltier effect to the efficiency of the thermoelement branch for different types of impurity distribution. To this end, numerical methods were used to solve the boundary value problem of the thermal balance in the branch of the thermoelectric element, taking into account the distributed Peltier effect. The case of nondegenerate charge carriers is considered in the framework of the standard two-band model. The parameters of the charge carriers were selected close to the thermoelectrics based on bismuth and antimony tellurides As shown by the calculation in the framework of the two-band model, the use of the distributed Peltier effect leads only to a partial absorption of the Joule heat, which contributes to an increase in the overall efficiency of the branch, while the $Z$ parameter along a significant part of the branch takes values significantly lower than the maximum value.

\section{Продолжение публикации материалов Конференции см. в No 2/22}

